\title{
SF3B1ness score: screening SF3B1 mutation status from over 60,000 transcriptomes based on a machine learning approach
}

\author{
Yuichi Shiraishi ${ }^{1,2,3^{*}}$, Kenichi Chiba ${ }^{1,2}$, Ai Okada $^{1,2}$ \\ 1. National Cancer Center Research Institute \\ 2. National Cancer Center Center for Cancer Genomics and Advanced Therapeutics \\ 3. RIKEN Center for Advanced Intelligence Project \\ *yuishira@ncc.go.jp
}

\section{Abstract}

In precision oncology, genomic evidence is used to determine the optimal treatment for each patient. However, identification of somatic mutations from genome sequencing data is often technically difficult and functional significance of somatic mutations is inconclusive in many cases. In this paper, to seek for an alternative approach, we tackle the problem of predicting functional mutations from transcriptome sequencing data. Focusing on SF3B1, a key splicing factor gene, we develop SF3B1ness score for classifying functional mutation status using a combination of Naive Bayes classifier and zero-inflated beta-binomial modeling ( $\mathrm{R}$ package is available at (https://github.com/friend1ws/SF3B1ness). Using 8,992 TCGA exome and RNA sequencing data for evaluation, we show that the classifier based on SF3B1ness score is able to (1) attain very high precision (>93\%) and sensitivity (>95\%), (2) rescue several somatic mutations not identified by exome sequence analysis especially due to low variant allele frequencies, and (3) successfully measure functional importance for somatic mutation whose significance has been unknown. Furthermore, to demonstrate that the SF3B1ness score is highly robust and can be extensible to the cohorts outside training data, we performed a functional SF3B1 mutation screening on 51,577

additional transcriptome sequencing data. We have detected 135 samples with putative SF3B1 functional mutations including those that are rarely registered in the somatic mutation database (e.g., G664C, L747W, and R775G). Moreover, we could identify two cases with SF3B1 mutations from normal tissues, implying that SF3B1ness score can be used for detecting clonal hematopoiesis.

\section{Introduction}

Advances in high throughput sequencing technology are bringing about revolutions in medicine. By sequencing each patient's cancer genomes, clinicians can discover appropriate treatment specifically targeted to the detected somatic variants. On the other hand, somatic mutations profiled via genome sequencing is usually far from perfect. It is still difficult to identify somatic mutations with complex forms [25, 27] and low variant allele frequencies 21]. In addition, although a vast number of cancer genome studies revealed a huge number of novel cancer-driving genes, the functional significance of variant for each position is still uninvestigated even for established cancer genes such as BRCA1/2 [2] and EGFR [1]. 
A

Evaluate SF3B1-ness classifier on 8,976 TCGA data via cross validation

C

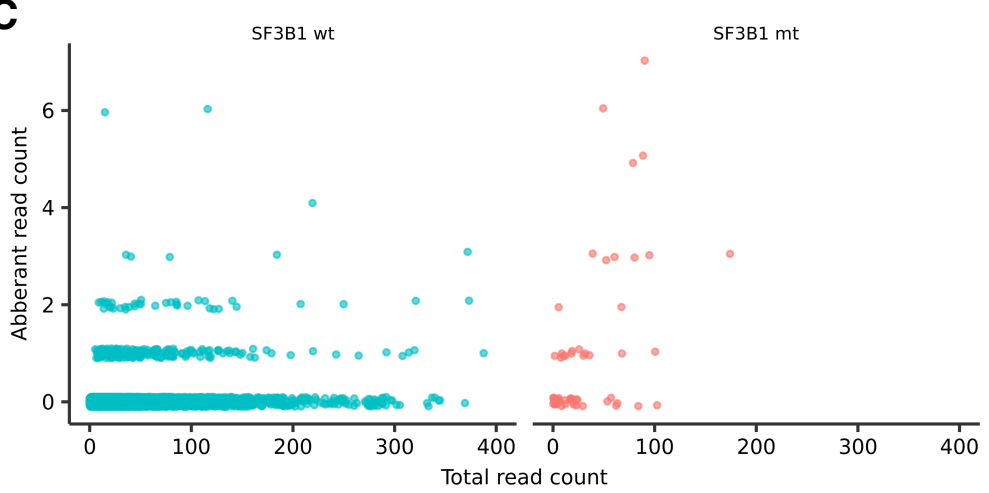

B

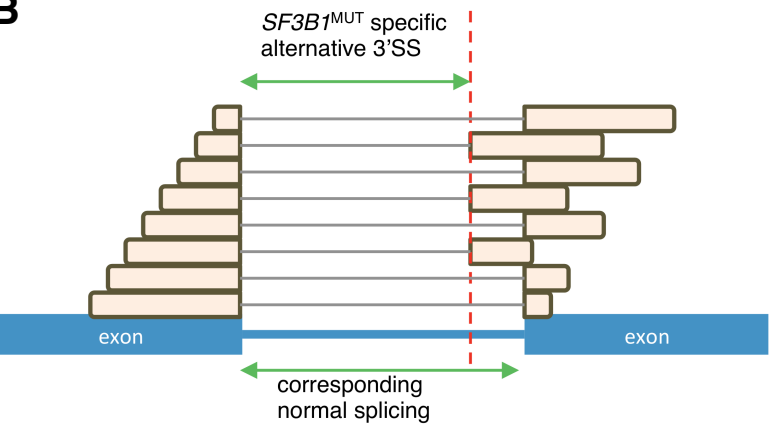

D

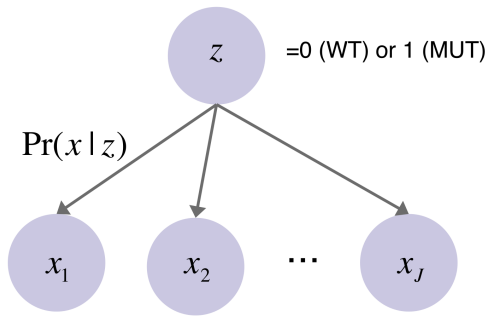

Figure 1. Overview of the study. A, The process of evaluating SF3B1ness classifier and screening functional SF3B1 mutations. B, Illustration of SF3B1 $1^{\mathrm{MUT}}$ associated alternative 3'SS and its corresponding normal splicing identified by split-aligned short reads. C, An example of supporting read counts of alternative 3'SS (chr1:100477090-100480840) and its corresponding normal splicing (chr1:100477090-100480857). Each point shows the counts of an individual. D, The generative model of assumed in this paper. The counts of SF3B1 $1^{\mathrm{MUT}}$ associated alternative 3'SS supporting reads are conditionally independent given the SF3B1 mutation status.

Using RNA sequencing together with genome sequencing often help complementing somatic mutation discovery and measuring the effect of a genomic mutation on transcriptomes such as expression and splicing changes. Here, we would tackle the problem of classifying "functional" mutation status based on transcriptome using statistical machine learning approaches.

In this paper, we would like to focus on SF3B1 gene, which encodes a core component of the RNA splicing machinery. SF3B1 is recurrently mutated in blood cancers 29 and other solid tumors 7,17 . The somatic mutations periodically cluster to positions within C-terminal HEAT repeat domains (HDs) with several major hotspots including p.R625, p.K666, p.K700 [20]. Although previous studies have clarified the functional significance for a few prominent positions [1, and the other sites that are less frequently but recurrently mutated are little investigated.

Figure 1A illustrates the outline of this study. We first develop a novel statistical approach that can effectively capture the characteristics of splicing changes induced by SF3B1 mutations using zero-inflated beta-binomial distribution naive Bayes classifier. Then, applying the proposed method to TCGA transcriptome sequencing data, we fit and evaluate the model on samples across 31 cancer types. Through that process, we provide a novel measure, SF3B1ness score, that quantifies transcriptional abnormalities caused by SF3B1 functional mutations in a unified way. Then, through the use of SF3B1ness score on about 60,000 publicly available transcriptome sequencing data, we 
perform large scale screening of SF3B1 functional mutations.

\section{Result}

\section{Method overview}

It has been known that functional SF3B1 mutations generate hundreds to thousands of genome-wide aberrant splicing events and these are mostly alternative 3'SS (splice site), where one end of splicing junction is at the annotated splicing donor site and the other end is distantly located from the annotated splicing acceptor site (see Figure 1B). Therefore, we first collected the set of these SF3B1 $1^{\text {MUT }}$ associated alternative 3'SS events from two previous studies [1.4], and use the quantification of these events (the numbers of short sequence reads spanning the target splicing junction [14,23]) as the features for the classification problem. In addition, the count of the corresponding normal splicing event for each alternative 3'SS is also used for measuring the ratio of alternative 3'SS (see Material Method for detail). In the end, we could extract 710 pairs of SF3B1 ${ }^{\mathrm{MUT}}$ associated alternative 3'SS and corresponding normal splicing.

Calculations of splicing junction events require downloading and alignment of raw sequencing data and thus needs considerable storage and computational cost. However, there is an excellent resource, the recount2 [3], which provides already well-processed transcriptome data including splicing junction counts for $\geq 70,000$ samples from $\geq$ 2,000 different studies including The Cancer Genome Atlas (TCGA) and The Genotype-Tissue Expression (GTEx) project. Therefore, to quantify SF3B1 ${ }^{\mathrm{MUT}}$ associated alternative 3'SS, we just downloaded the splicing junction data from the recount2 for each study and extracted those matching the SF3B1 ${ }^{\mathrm{MUT}}$ associated alternative 3'SS and their corresponding normal splicing.

For typical SF3B1 $1^{\mathrm{MUT}}$ associated alternative 3'SS loci, aberrant read counts are zeros in most $S F 3 B 1^{\mathrm{WT}}$ samples, whereas some samples have a decent amount of aberrant read counts (see Figure 1C). Even though the ratios of aberrant read counts significantly increase for many SF3B1 $1^{\mathrm{MUT}}$ samples, still a certain number of samples have zero counts. In fact, these excess zero-count situations cannot be not effectively captured by common probabilistic distributions such as binomial distribution, and zero-inflated models (mixtures of probability mass at zero and other distributions) have been used in many biological data analysis such as single-cell transcriptome 10,19 and microbiome 8, 18, 26. In this paper, we assume that the counts of $S F 3 B 1^{\mathrm{MU}}$ associated alternative 3'SS and corresponding normal splicing are generated by zero-inflated beta-binomial distribution (see Material Method for detail).

For constructing the classifier on SF3B1 mutation status from alternative 3'SS count data, we adopt naive Bayes classifier (see Figure 1D), The detailed procedure is as follows (see Material Method for detail):

1. We divide the samples into $S F 3 B 1^{\mathrm{WT}}$ and $S F 3 B 1^{\mathrm{MUT}}$ groups. Then, for each group and each $S F 3 B 1^{\mathrm{MUT}}$ associated alternative 3 'SS, we estimate the parameters of zero-inflated beta-binomial model.

2. Assuming the conditional independence among the $S F 3 B 1^{\mathrm{MUT}}$ associated alternative 3'SS events, we calculate the posterior probabilities of $S F 3 B 1^{\mathrm{WT}}$ and SF3B1 $1^{\mathrm{MUT}}$ based on the parameters estimated above

3. By taking the difference of the log probabilities of $S F 3 B 1^{\mathrm{MUT}}$, which we define as "SF3B1ness" score, we predict the SF3B1 mutation status (positive if and only if the SF3B1ness score is above a threshold). 


\section{Application to TCGA data}

To evaluate the effectiveness of SF3B1ness classifier, we applied the proposed approach to TCGA data set. We selected the set of 8,992 primary cancer samples from 31 types and determined the somatic mutation status for SF3B1 as described in the previous study [23]. In this section, referring to past literature, we consider the SF3B1 hotspot mutations as those occurring at E622, R625, N626, H662, K666, K700, G740, K741, G742. Then, there are 63 samples with SF3B1 hotspot mutations in total. The cancer types with frequent SF3B1 hotspot mutations are breast invasive carcinoma (BRCA, 14 / 826), skin cutaneous melanoma (SKCM, 8 / 469), and uveal melanoma (UVM, 17 / 80). Besides, there are other cancer types with SF3B1 hotspot mutations albeit the frequencies is low including bladder urothelial carcinoma, kidney renal clear cell carcinoma, lung adenocarcinoma, prostate adenocarcinoma, and so on.

We divided 8,992 samples into two datasets and used one dataset (training data) for fitting the parameters of SF3B1ness classifier and obtained the SF3B1ness score for each sample in the other data set (test data). Then, we performed the same procedure exchanging the training and test data. When training the parameters, samples with well known SF3B1 hotspot are categorized as positive cases $\left(z_{i}=1\right)$, and those without any SF3B1 mutations are categorized as negative cases $\left(z_{i}=0\right)$. Note that samples with non-hotspot SF3B1 mutations are removed in the training phases because these biological functions remain to be investigated and only used in test phases. Importantly, we used samples from all the 31 cancer types together without any distinction. Although the amount of expression of each SF3B1 ${ }^{\text {MUT }}$ associated alternative 3'SS varied according to cancer types, we believe that our model is sufficiently robust to these variations. The key to this robustness is that we take not only the absolute count of SF3B1 ${ }^{\text {MUT }}$ associated alternative 3'SS but also the fraction to its normal splicing into account via beta-binomial distribution. Furthermore, the adoption of zero-inflated components makes the probabilistic model tolerant of other disturbing factors such as outliers and mislabeling.

First, the mutation status could be very accurately predicted by the proposed method. Most of the samples with high SF3B1ness score actually had SF3B1 hotspot mutations (see Figure 2A and S1 Table). Among 63 samples with known SF3B1 hotspot mutations, 60 samples had positive SF3B1ness score, indicating that the sensitivity of SF3B1 score is more than $95 \%$.

There were 13 samples with positive SF3B1 score even without hotspot SF3B1 mutations (see Table 1). Those samples are categorized into the following three classes.

1. Four samples had non-hotspot SF3B1 mutations (such as T663P, Q699E, R775L, and D781E). Although many of them have been comparably rarely detected in cancer genome studies, decent amounts of SF3B1ness score suggest that they have similar functions to hotspot mutations on genome-wide splicing aberrations.

2. For other four samples, after manually investigating the exome and RNA sequencing data, we could detect a number of short reads supporting SF3B1 hotspot somatic mutation even though the variant allele frequencies are very low $(1 \%-10 \%)$. Therefore, even with low variant allele frequencies, the function of SF3B1 somatic mutation can be detectable.

3. For the remaining five samples, we could not identify any SF3B1 putative functional mutations after extensive manual investigations. Still, the profiles of alternative 3'SS counts for these samples are very similar to those with hotspot SF3B1 mutations (see Figure 2C), implying that there may be other genomic mutations on the same pathway with SF3B1 concealed in these samples. 

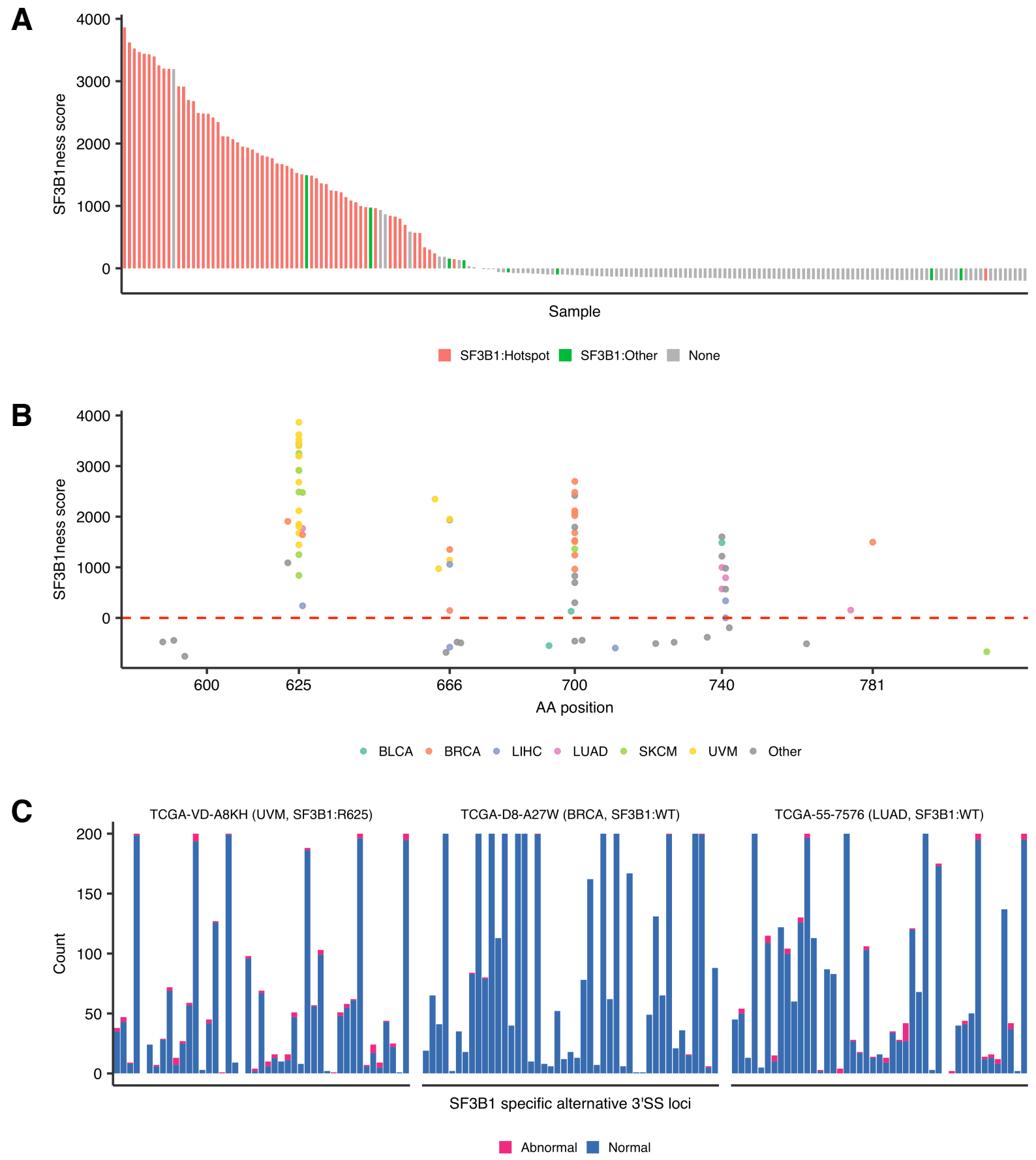

Figure 2. The overview of SF3B1ness score evaluation on TCGA data. A, The relationships between SF3B1ness scores and SF3B1 mutation status. Each bar shows each individual from TCGA dataset, and the color shows SF3B1 mutation status (hotspot are defined in the above). B, The relationships between SF3B1ness scores and amino-acids positions for samples with SF3B1 mutations. The color shows the cancer types. C, The counts of SF3B1 ${ }^{\mathrm{MUT}}$ associated alternative 3'SS and its corresponding normal splicing junction for several characteristic samples. Each bar shows the loci of SF3B1 ${ }^{\mathrm{MUT}}$ associated alternative 3'SS. For loci with $\geq 200$ supporting read counts in total, they are down-sampled to 200. 
Table 1. List of samples with high SF3B1ness score and without hotspot SF3B1 mutations.

\begin{tabular}{|c|c|c|c|}
\hline Sample name & Cancer type & Score & Remark \\
\hline 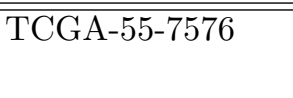 & 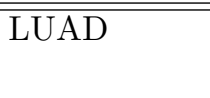 & 3193.496 & $\begin{array}{l}\text { No SF3B1 mutation is identified after manual investigations. } \\
\text { (low variant allele frequency). }\end{array}$ \\
\hline TCGA-E2-A10F & BRCA & 1495.878 & D781E mutation is identified by usual exome data analysis. \\
\hline TCGA-V4-A9EC & UVM & 972.768 & T663P mutation is identified by usual exome data analysis. \\
\hline TCGA-3H-AB3K & MESO & 935.228 & No SF3B1 mutation is identified after manual investigations. \\
\hline TCGA-XM-A8RI & THYM & 866.908 & $\begin{array}{l}\text { K700E mutation is identified after manual investigations. } \\
\text { (low variant allele frequency). }\end{array}$ \\
\hline TCGA-05-4432 & LUAD & 586.704 & No SF3B1 mutation is identified after manual investigations. \\
\hline TCGA-75-5125 & LUAD & 190.175 & $\begin{array}{l}\text { K700E mutation is identified after manual investigations. } \\
\text { (low variant allele frequency). }\end{array}$ \\
\hline TCGA-ZP-A9CV & LIHC & 186.524 & No SF3B1 mutation is identified after manual investigations. \\
\hline TCGA-NJ-A55O & LUAD & 153.664 & R775L mutation is identified by usual exome data analysis. \\
\hline TCGA-NJ-A4YQ & LUAD & 136.287 & No SF3B1 mutation is identified after manual investigations. \\
\hline TCGA-UY-A78N & BLCA & 130.962 & Q699E mutation is identified by usual exome data analysis. \\
\hline TCGA-EY-A2OM & UCEC & 38.213 & No SF3B1 mutation is identified after manual investigations. \\
\hline TCGA-WC-A87W & UVM & 21.388 & $\begin{array}{l}\mathrm{R} 625 \mathrm{H} \text { mutation is identified after manual investigations. } \\
\text { (low variant allele frequency). }\end{array}$ \\
\hline
\end{tabular}

The SF3B1 somatic mutations linked to high SF3B1ness scores are in the proximity of known hotspots (see Figure 2B). Although this is anticipated by the marked concentration of SF3B1 mutations and oncogenic functions thereof. Still, SF3B1ness score discerned the amino acid positions into those with high (e.g., T663P, Q699E) from those with low (e.g., I665F, V668I, G693) scores even among the proximity of hotspots.

Collectively, SF3B1ness score is helpful for measuring the effects of rare SF3B1 mutations as well as saving mutations misidentified because of some technical problems such as low variant allele frequencies. Assuming that samples belonging to the first two classes are true positives, SF3B1ness score has fairly high precision $(93.15 \%)$ on the functional SF3B1 mutation status prediction.

\section{Screening of SF3B1 functional mutations from large scale transcriptome resource}

To evaluate the ability of SF3B1ness score on dataset not seen during training, we performed further large scale screening of functional SF3B1 mutation from in total of 51,577 transcriptome data in the recount2 resource, where we removed data with small sizes (with $\leq 100 \mathrm{M}$ bases). We first calculated SF3B1ness score just by downloading splicing junction data (which is far more small in size than raw sequencing data). Then, for the samples with high SF3B1ness scores, we downloaded their raw sequence data, aligned to the reference genome and checked the SF3B1 mutation status.

First, we identified 154 samples with $\geq 50.0$ SF3B1ness scores. Of those, we could identify 140 samples having SF3B1 mutations registered in the COSMIC database within C-terminal HEAT repeat domains (residues 604-801). When narrowing down the 124 samples with $\geq 300.0$ SF3B1ness scores, then 123 samples $(123 / 124=0.992)$ had COSMIC registered SF3B1 mutations, indicating that the SF3B1ness score is highly robust and can be utilized to a broad type of transcriptome data without caring tissue or cancer types (see Figure 3A and S2 Table). 

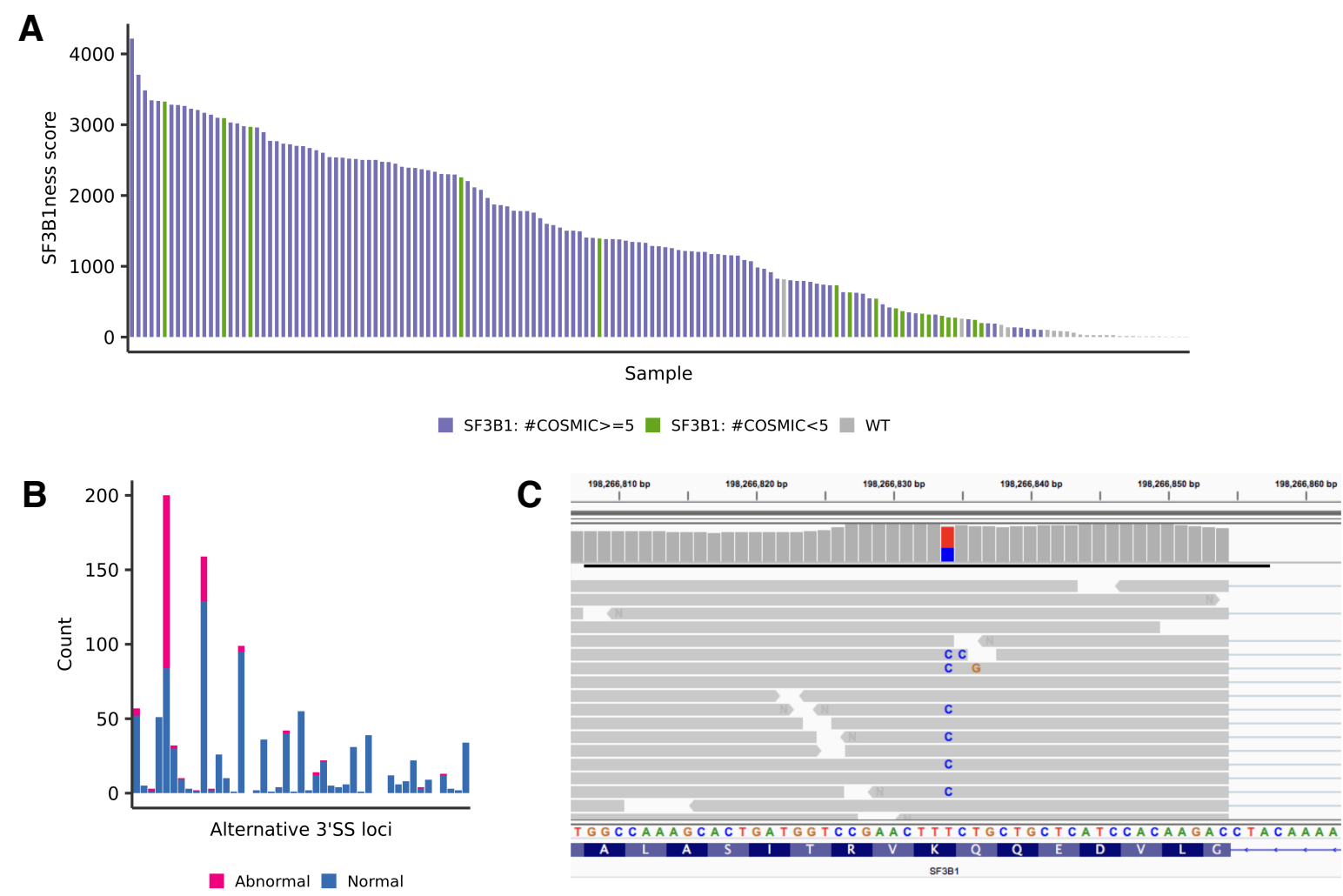

Figure 3. Overview of SF3B1 functional screening using the recount2. A, The relationships between SF3B1ness scores and SF3B1 mutation status. Each bar shows each individual from recount2 dataset, and the color shows SF3B1 mutation status (COSMIC release v87 is used for the registered number of SF3B1 mutations). B, C, The counts of SF3B1 ${ }^{\mathrm{MUT}}$ associated alternative 3'SS and its corresponding normal splicing junction (B) and the alignment view around SF3B1 K700E for the sample having high SF3B1ness score (SRR1374647) in GTEx study (C).

Many of the SF3B1 mutations found in high SF3B1ness score samples are hotspot mutations (a large number of records registered in COSMIC database). However, there are several substitutions (R625G, G664C, K741N, L747W, and R775G) with few records in COSMIC, suggesting the functional importance of these rare mutations and awaiting for further biological experiments.

Most samples with high SF3B1ness scores are found in cancer types where SF3B1 mutations are known to be prevalent, including AML (acute myeloid leukemia), CLL (chronic lymphocytic leukemia), MDS (myelodysplastic syndrome), breast cancer, melanoma, and uveal melanoma. However, we could also find SF3B1 mutations from two normal samples; one is from a whole blood sample (SRR1374647,

GTEX-ZF28-0005-SM-4WKH3) from GTEx study [15] (see Figure 3B,C). The other is from a bone sample from an old woman (SRR2305491) 6]. These facts indicate that SF3B1 mutations induce splicing alterations in even normal tissues and SF3B1ness score may be helpful for efficient detection of clonal hematopoiesis from individuals without apparent hematological malignancies.

\section{Discussion}

In this paper, we could constitute the classifier, SF3B1ness score, to accurately identify SF3B1 functional mutation status. Most classifiers for predicting some pathway 
activation are trained on specific cancer types or tissues. However, the SF3B1ness score developed in this paper is highly robust so that users can easily apply it to their own data without caring the properties target transcriptome sequencing data. This will facilitate the application of SF3B1ness score for screening SF3B1 mutation status from large scale transcriptome data. The robustness is partly because the target loci of splicing changes by SF3B1 mutations are mostly common across cancer types and tissues. However, at the same time, the delicately designed probabilistic model (e.g., adopting zero-inflation components) significantly contribute to this robustness.

There have been active researches on inhibiting SF3B1 as a therapeutic target [13, and several studies demonstrated that mutations within SF3B1 impact on the sensitivities to SF3B1 inhibitors 16,28, suggesting that the SF3B1ness proposed in this study may be helpful to evaluate the activity of aberrant splicing, and future precision medicine.

One may argue that directly investigating SF3B1 mutation by aligning transcriptome sequencing data and checking the variant allele frequencies of known hotspots is much easier and more straightforward. For that opinion, we would like to insist that automatically detecting somatic mutation is still not trivial especially for those with low variant allele frequencies and our approach can give another way to confirm and rescue SF3B1 functional mutations. In addition, since raw sequencing data usually includes information that is sufficient to identify individuals and we need to follow ethically appropriate procedures (which is often time-consuming) to access and manage raw sequencing data. On the other hand, several consortium and resource distribute already pre-processed splicing junction data $3,9,12,15]$, where our approach offers perfectly simple and accurate way for somatic mutation screening.

There are other splicing factor genes such as U2AF1, SRSF2, and several studies show that SETD2, a histone methyltransferase, is also related to aberrant splicing [24]. In fact, the characteristics of splicing changes in these genes are largely different. For SF3B1 mutation, completely novel splicing junctions are generated, whereas just the ratios of already splicing junctions change by the mutation in other genes. Therefore, to be able to constitute classifier for other genes related to splicing, the model developed in this paper need to be significantly refined.

\section{Materials and Methods}

\section{Collection of SF3B1 ${ }^{\mathrm{MUT}}$ associated alternative 3'SS}

Through a large number of studies $1,4,4,5,22$, the characteristics of SF3B1 ${ }^{\mathrm{MUT}}$ abnormal splicing are known as follows:

- These aberrant splicing events are mostly alternative 3'SS.

- Also, the new acceptor sites of SF3B1 ${ }^{\mathrm{MUT}}$ associated alternative 3'SS events are typically located within 18 to 50bp downstream from the authentic acceptor sites.

We have compiled $S F 3 B 1^{\mathrm{MUT}}$ specific abnormal splicing junctions from two previous studies. The first set is 895 splicing junctions identified by comparing 35 SF3B1 $1^{\text {MUT }}$ and $50 S F 3 B 1^{\mathrm{WT}}$ samples from chronic lymphocytic leukemia, breast cancer, skin melanoma and uveal melanoma [4]. The second set is 1,124 spicing junctions identified by comparing $16 S F 3 B 1^{\mathrm{MUT}}$ and $56 S F 3 B 1^{\mathrm{WT}}$ samples from uveal melanoma 1 . We gathered the splicing junctions from the two studies and removed splicing junctions matching with annotated introns defined by Gencode Version 19 (http://hgdownload. cse.ucsc.edu/goldenPath/hg19/database/wgEncodeGencodeBasicV19.txt.gz).

Furthermore, we request that one end of splicing junction is at the annotated splicing 
donor site and the other is located within 50bp upstream of canonical splicing acceptor sites. Then, the remaining the coordinates of splicing junctions are converted to GRCh38 based positions.

\section{A generative model of splicing junction counts via zero-inflated beta-binomial distribution}

Let $x_{i, j}, y_{i, j}$ denote the counts of $j$-th SF3B1 ${ }^{\mathrm{MUT}}$ associated alternative 3'SS and the corresponding normal splicing for the $i$-th sample $(i=1,2, \cdots, I, j=1,2, \cdots, J)$. Setting $n_{i, j}=x_{i, j}+y_{i, j}$, then the probability function of $x_{i, j}$ is

$$
\begin{gathered}
f\left(x_{i, j} \mid n_{i, j}, \alpha_{j}, \beta_{j}, \pi_{j}\right)=\left(1-\pi_{j}\right) g\left(x_{i, j} \mid n_{i, j}, \alpha_{j}, \beta_{j}\right),\left(x_{i, j}>0\right), \\
f\left(x_{i, j} \mid n_{i, j}, \alpha_{j}, \beta_{j}, \pi_{j}\right)=\pi_{j}+\left(1-\pi_{j}\right) g\left(x_{i, j}=0 \mid n_{i, j}, \alpha_{j}, \beta_{j}\right),\left(x_{i, j}=0\right)
\end{gathered}
$$

where $g$ is the beta-binomial density function,

$$
\begin{aligned}
& g\left(x_{i, j} \mid n_{i, j}, \alpha_{j}, \beta_{j}\right) \\
= & \frac{\Gamma\left(n_{i, j}+1\right)}{\Gamma\left(x_{i, j}+1\right) \Gamma\left(n_{i, j}-x_{i, j}+1\right)} \frac{\Gamma\left(x_{i, j}+\alpha_{j}\right) \Gamma\left(n_{i, j}-x_{i, j}+\beta_{j}\right)}{\Gamma\left(n_{i, j}+\alpha_{j}+\beta_{j}\right)} \frac{\Gamma\left(\alpha_{j}+\beta_{j}\right)}{\Gamma\left(\alpha_{j}\right) \Gamma\left(\beta_{j}\right)} .
\end{aligned}
$$

The parameters $\alpha_{j}, \beta_{j}, \pi_{j}$ are estimated by numerically maximizing the log-likelihood,

$$
l_{j}\left(\alpha_{j}, \beta_{j}, \pi_{j}\right)=\prod_{i=1}^{I} \log f\left(x_{i, j} \mid n_{i, j}, \alpha_{j}, \beta_{j}, \pi_{j}\right) .
$$

\section{A classification model for $S F 3 B 1$ mutation status using naive Bayes model}

Suppose $z_{i} \in\{0,1\}$ is the $S F 3 B 1$ mutation status for the $i$-th sample (0:SF3B1 ${ }^{\mathrm{WT}}, 1$ : $\left.S F 3 B 1^{\mathrm{MUT}}\right)$. First, for each $S F 3 B 1^{\mathrm{MUT}}$ associated alternative 3'SS, we estimate the parameters of zero-inflated beta-binomial distribution for $S F 3 B 1^{\mathrm{WT}}$ and $S F 3 B 1^{\mathrm{MUT}}$ groups. Let $\hat{\boldsymbol{\alpha}}^{\mathbf{0}}=\left(\hat{\alpha}_{j}^{0}\right)_{j=1, \cdots, J}, \hat{\boldsymbol{\beta}}^{\mathbf{0}}=\left(\hat{\beta}_{j}^{0}\right)_{j=1, \cdots, J}, \hat{\boldsymbol{\pi}}^{\mathbf{0}}=\left(\hat{\pi}_{j}^{0}\right)_{j=1, \cdots, J}$ denote the parameters estimated for SF3B1 ${ }^{\mathrm{WT}}$ groups and $\hat{\boldsymbol{\alpha}}^{\mathbf{1}}=\left(\hat{\alpha}_{j}^{1}\right)_{j=1, \cdots, J}, \hat{\boldsymbol{\alpha}}^{\mathbf{1}}=\left(\hat{\alpha}_{j}^{1}\right)_{j=1, \cdots, J}$, $\hat{\pi}^{1}=\left(\hat{\pi}_{j}^{1}\right)_{j=1, \cdots, J}$ the parameters for $S F 3 B 1^{\mathrm{MUT}}$ groups, respectively.

Then, by applying Bayes' theorem, the conditional probabilities are

$$
\operatorname{Pr}\left(z_{i} \mid \boldsymbol{x}_{i}, \boldsymbol{n}_{i}\right) \propto \operatorname{Pr}\left(z_{i}\right) \prod_{j=1}^{J} \operatorname{Pr}\left(x_{i, j} \mid z_{i}, n_{i, j}\right) .
$$

Therefore,

$$
\begin{gathered}
\operatorname{Pr}\left(z_{i}=0 \mid \boldsymbol{x}_{i}, \boldsymbol{n}_{i}\right) \propto \gamma^{0} \prod_{j=1}^{J} f\left(x_{i, j} \mid n_{i, j}, \hat{\alpha}_{j}^{0}, \hat{\beta}_{j}^{0}, \hat{\pi}_{j}^{0}\right), \\
\operatorname{Pr}\left(z_{i}=1 \mid \boldsymbol{x}_{i}, \boldsymbol{n}_{i}\right) \propto\left(1-\gamma^{0}\right) \prod_{j=1}^{J} f\left(x_{i, j} \mid n_{i, j}, \hat{\alpha}_{j}^{1}, \hat{\beta}_{j}^{1}, \hat{\pi}_{j}^{1}\right),
\end{gathered}
$$

where $\gamma^{0}$ is the parameter corresponding to $\operatorname{Pr}\left(z_{i}=0\right)$ (in this paper, we adopt non-informative value $\gamma^{0}=1 / 2$ ). Finally, for each new sample, we evaluate the logarithm of the ratio of conditional probabilities (SF3B1ness score)

$$
\log \operatorname{Pr}\left(z_{i}=1 \mid \boldsymbol{x}_{i}, \boldsymbol{n}_{i}\right)-\log \operatorname{Pr}\left(z_{i}=0 \mid \boldsymbol{x}_{i}, \boldsymbol{n}_{i}\right)
$$




\section{Supporting Information}

\section{S1 Table.}

The SF3B1ness scores and mutation status for each TCGA samples.

\section{S2 Table.}

The SF3B1ness scores and mutation status for each recount2 samples with high SF3B1ness scores. In the Mutation_Info columns, the amino-acids changes, the numbers of registered mutations in COSMIC database and variant allele frequencies are concatenated by commas. Also, if there are multiple somatic SF3B1 mutations, these strings are linked together by semi-colons.

\section{Acknowledgments}

The authors initiated this research project inspired by discussions with Dr. Claus Thorn Ekstrøm and Dr. Xiang Zhou through "No disease is an island" project, which is financed by the Danish Agency for Science and Higher Education. We would like to thank them.

\section{References}

1. S. Alsafadi, A. Houy, A. Battistella, T. Popova, M. Wassef, E. Henry, F. Tirode, A. Constantinou, S. Piperno-Neumann, S. Roman-Roman, et al. Cancer-associated SF3B1 mutations affect alternative splicing by promoting alternative branchpoint usage. Nature communications, 7:10615, 2016.

2. J. Y. Cheon, J. Mozersky, and R. Cook-Deegan. Variants of uncertain significance in BRCA: a harbinger of ethical and policy issues to come? Genome medicine, 6(12):121, 2014.

3. L. Collado-Torres, A. Nellore, K. Kammers, S. E. Ellis, M. A. Taub, K. D. Hansen, A. E. Jaffe, B. Langmead, and J. T. Leek. Reproducible RNA-seq analysis using recount2. Nature biotechnology, 35(4):319, 2017.

4. R. B. Darman, M. Seiler, A. A. Agrawal, K. H. Lim, S. Peng, D. Aird, S. L. Bailey, E. B. Bhavsar, B. Chan, S. Colla, et al. Cancer-associated SF3B1 hotspot mutations induce cryptic 3' splice site selection through use of a different branch point. Cell reports, 13(5):1033-1045, 2015.

5. C. DeBoever, E. M. Ghia, P. J. Shepard, L. Rassenti, C. L. Barrett, K. Jepsen, C. H. Jamieson, D. Carson, T. J. Kipps, and K. A. Frazer. Transcriptome sequencing reveals potential mechanism of cryptic 3'splice site selection in SF3B1-mutated cancers. PLoS computational biology, 11(3):e1004105, 2015.

6. J. N. Farr, M. M. Roforth, K. Fujita, K. M. Nicks, J. M. Cunningham, E. J. Atkinson, T. M. Therneau, L. K. McCready, J. M. Peterson, M. T. Drake, et al. Effects of age and estrogen on skeletal gene expression in humans as assessed by RNA sequencing. PLoS One, 10(9):e0138347, 2015.

7. J. W. Harbour, E. D. Roberson, H. Anbunathan, M. D. Onken, L. A. Worley, and A. M. Bowcock. Recurrent mutations at codon 625 of the splicing factor SF3B1 in uveal melanoma. Nature genetics, 45(2):133, 2013. 
8. T. Hu, P. Gallins, and Y.-H. Zhou. A zero-inflated beta-binomial model for microbiome data analysis. Stat, page e185, 2018.

9. M. A. Jensen, V. Ferretti, R. L. Grossman, and L. M. Staudt. The NCI genomic data commons as an engine for precision medicine. Blood, 130(4):453-459, 2017.

10. P. V. Kharchenko, L. Silberstein, and D. T. Scadden. Bayesian approach to single-cell differential expression analysis. Nature methods, 11(7):740, 2014.

11. S. Kohsaka, M. Nagano, T. Ueno, Y. Suehara, T. Hayashi, N. Shimada, K. Takahashi, K. Suzuki, K. Takamochi, F. Takahashi, et al. A method of high-throughput functional evaluation of EGFR gene variants of unknown significance in cancer. Science translational medicine, 9(416):eaan6566, 2017.

12. A. Lachmann, D. Torre, A. B. Keenan, K. M. Jagodnik, H. J. Lee, L. Wang, M. C. Silverstein, and A. Ma'ayan. Massive mining of publicly available RNA-seq data from human and mouse. Nature communications, 9(1):1366, 2018.

13. S. C.-W. Lee and O. Abdel-Wahab. Therapeutic targeting of splicing in cancer. Nature medicine, 22(9):976, 2016.

14. Y. I. Li, D. A. Knowles, J. Humphrey, A. N. Barbeira, S. P. Dickinson, H. K. Im, and J. K. Pritchard. Annotation-free quantification of RNA splicing using LeafCutter. Nature genetics, 50(1):151, 2018.

15. J. Lonsdale, J. Thomas, M. Salvatore, R. Phillips, E. Lo, S. Shad, R. Hasz, G. Walters, F. Garcia, N. Young, et al. The genotype-tissue expression (GTEx) project. Nature genetics, 45(6):580, 2013.

16. S. L. Maguire, A. Leonidou, P. Wai, C. Marchiò, C. K. Ng, A. Sapino, A.-V. Salomon, J. S. Reis-Filho, B. Weigelt, and R. C. Natrajan. SF3B1 mutations constitute a novel therapeutic target in breast cancer. The Journal of pathology, 235(4):571-580, 2015.

17. C. G. A. Network et al. Comprehensive molecular portraits of human breast tumours. Nature, 490(7418):61, 2012.

18. J. N. Paulson, O. C. Stine, H. C. Bravo, and M. Pop. Differential abundance analysis for microbial marker-gene surveys. Nature methods, 10(12):1200, 2013.

19. E. Pierson and C. Yau. ZIFA: Dimensionality reduction for zero-inflated single-cell gene expression analysis. Genome biology, 16(1):241, 2015.

20. M. Seiler, S. Peng, A. A. Agrawal, J. Palacino, T. Teng, P. Zhu, P. G. Smith, S. J. Caesar-Johnson, J. A. Demchok, I. Felau, et al. Somatic mutational landscape of splicing factor genes and their functional consequences across 33 cancer types. Cell reports, 23(1):282-296, 2018.

21. H.-T. Shin, Y.-L. Choi, J. W. Yun, N. K. Kim, S.-Y. Kim, H. J. Jeon, J.-Y. Nam, C. Lee, D. Ryu, S. C. Kim, et al. Prevalence and detection of low-allele-fraction variants in clinical cancer samples. Nature Communications, 8(1):1377, 2017.

22. Y. Shiozawa, L. Malcovati, A. Gallì, A. Sato-Otsubo, K. Kataoka, Y. Sato, Y. Watatani, H. Suzuki, T. Yoshizato, K. Yoshida, et al. Aberrant splicing and defective mRNA production induced by somatic spliceosome mutations in myelodysplasia. Nature communications, 9(1):3649, 2018. 
23. Y. Shiraishi, K. Kataoka, K. Chiba, A. Okada, Y. Kogure, H. Tanaka, S. Ogawa, and S. Miyano. A comprehensive characterization of cis-acting splicing-associated variants in human cancer. Genome research, 28(8):1111-1125, 2018.

24. J. M. Simon, K. E. Hacker, D. Singh, A. R. Brannon, J. S. Parker, M. Weiser, T. H. Ho, P.-F. Kuan, E. Jonasch, T. S. Furey, et al. Variation in chromatin accessibility in human kidney cancer links h3k36 methyltransferase loss with widespread rna processing defects. Genome research, 24(2):241-250, 2014.

25. J. A. Wala, P. Bandopadhayay, N. Greenwald, R. O'Rourke, T. Sharpe, C. Stewart, S. Schumacher, Y. Li, J. Weischenfeldt, X. Yao, et al. SvABA: genome-wide detection of structural variants and indels by local assembly. Genome research, 2018.

26. L. Xu, A. D. Paterson, W. Turpin, and W. Xu. Assessment and selection of competing models for zero-inflated microbiome data. PloS one, 10(7):e0129606, 2015.

27. K. Ye, J. Wang, R. Jayasinghe, E.-W. Lameijer, J. F. McMichael, J. Ning, M. D. McLellan, M. Xie, S. Cao, V. Yellapantula, et al. Systematic discovery of complex insertions and deletions in human cancers. Nature medicine, 22(1):97, 2016.

28. A. Yokoi, Y. Kotake, K. Takahashi, T. Kadowaki, Y. Matsumoto, Y. Minoshima, N. H. Sugi, K. Sagane, M. Hamaguchi, M. Iwata, et al. Biological validation that SF3b is a target of the antitumor macrolide pladienolide. The FEBS journal, 278(24):4870-4880, 2011.

29. K. Yoshida, M. Sanada, Y. Shiraishi, D. Nowak, Y. Nagata, R. Yamamoto, Y. Sato, A. Sato-Otsubo, A. Kon, M. Nagasaki, et al. Frequent pathway mutations of splicing machinery in myelodysplasia. Nature, 478(7367):64, 2011. 